\title{
DISTRIBUIÇÃO ESPACIAL DO CONSUMO RESIDENCIAL DE ÁGUA TRATADA NA CIDADE DE UBERLÂNDIA-MG: ESTUDO DE CASOS PARA OS MESES DO ANO DE 2015.
}

\author{
Matheus Ferreira do Amaral \\ Universidade Federal de Uberlândia, Faculdade de Matemática \\ Graduação em Estatística, Uberlândia, MG, Brasil \\ matheusamaral.ferreira@hotmail.com \\ Fernanda Endo Faleiros \\ Universidade Federal de Uberlândia, Instituto de Ciências Agrárias \\ Pós-Graduação em Qualidade Ambiental, Uberlândia, MG, Brasil \\ fefalleiros@hotmail.com \\ Ednaldo Carvalho Guimarães \\ Universidade Federal de Uberlândia, Faculdade de Matemática \\ Uberlândia, MG, Brasil \\ ecg@ufu.br
}

\section{RESUMO}

A água é um recurso natural responsável pela origem da vida e sua sustentação no nosso planeta Terra. É considerada fundamental para o exercício das atividades econômicas como a agricultura e a indústria, exercendo também importância crucial na qualidade de vida das populações, especialmente, no que se refere ao abastecimento de água e a coleta e tratamento de esgotos, que têm forte impacto sobre a saúde pública. Assim para um melhor controle desse recurso, é necessária uma maior compreensão espacial e temporal do consumo. Neste sentido, o objetivo deste trabalho foi estudar o comportamento espacial do consumo médio diário de água potável na cidade de Uberlândia-MG, ao longo dos meses, para o ano de 2015. A análise da dependência espacial foi feita por meio dos semivariogramas, que foram utilizados na interpolação por krigagem e no mapeamento do consumo residencial de água. Os resultados permitiram concluir que a variável estudada, apresenta dependência espacial; o modelo que melhor se ajustou aos dados pertence a família Gaussiana, com um grau moderado de dependência; em relação a distribuição espacial, observa-se predominância de maior consumo de água residencial na região central da figura representativa da área de estudo.

PALAVRAS-CHAVE: Água tratada. Dependência espacial. Semivariograma.Geoestatística.

\section{SPATIAL DISTRIBUTION OF TREATED RESIDENTIAL WATER CONSUMPTION IN UBERLÂNDIA-MG CITY: STUDY OF CASES FOR THE MONTHS OF 2015 YEAR.}

\begin{abstract}
Water is a natural resource responsible for the origin of life and your support on our Earth planet. It's fundamental considered for the economic activities exercise such as agriculture and industry, also doing crucial importance of population life quality, especially, in what it mentions to water supply and the sewer treatment and collection, which has strong impact over the public health. So for a better control of this resource, is necessary a bigger spatial understanding and temporal of consuming. In this way, the objective of this article was study spatial behavior of the daily middle consumption of potable water in Uberlândia- MG city along the months, for the 2015 year. The spatial dependence analysis was made through semivariogram, which were used in the interpolation by kriging and consumption mapping of residential water. The results allowed to conclude that the variable studied, shows spatial dependence; the model which fits better to the data belongs to Gaussiana family, with an average level of dependence; in relation of spatial distribution, it is observed predominance of bigger residential water consumption in the central area of the representative figure from the study area.
\end{abstract}

Keywords: Treated water; Spatial dependence; semivariogram; Geostatistics 


\section{INTRODUÇÃO}

A água é recurso natural que exerce papel fundamental no desenvolvimento da vida e está associada a todas as atividades humanas. Entretanto ressalta-se que sua distribuição no planeta e sua disponibilidade para a população não é uniforme, sendo que existe uma tendência de agravamento de crises hídricas no planeta.

A crise hídrica resulta de um conjunto de componentes de origem social, econômica e ambiental, é um assunto em pauta, por limitar as condições de vida de grande parte da população mundial. Por muito tempo esta que foi considerada um recurso natural infinito, de pouco ou nenhum valor econômico teve no seu uso perdulário um dos principais motivos geradores da redução em sua oferta (HESPANHOL, 1999). Atualmente se debate sobre a gestão dos recursos hídricos e a necessidade de melhor gerenciamento desse recurso no que se refere à quantidade e qualidade, havendo uma necessidade de repensar a atual utilização da água, bem como criar novos comportamentos e atitudes que contribuam para a minimização dos problemas relacionados a esse recurso. Para contribuir com o conhecimento do comportamento do uso da água e, consequentemente, para um melhor gerenciamento desse recurso pode-se utilizar os modelos e técnicas estatísticas de análise de dados.

Pinto et al. (2015), apontam que apesar da grande quantidade de água existente no planeta somente uma pequena parcela está facilmente acessível e própria para o consumo, estando localizado nos rios, equivalendo a $0,3 \%$, dos $2,5 \%$ de água doce presente na superfície terrestre. Portanto, o planejamento de consumo e o uso racional da água devem ser pensados em qualquer setor da sociedade.

No atual momento em que muito se debate sobre a questão hídrica, visando à formulação de soluções para problemas como, por exemplo, a falta de abastecimento de água tratada para o consumo humano, estudos relacionados a compreensão da distribuição espacial do consumo dos recursos hídricos são pertinentes.

Araújo et al. (2009) enfatizaram que o estudo de variabilidade espacial, por meio da geoestatística, tem se mostrado de grande utilidade em várias áreas do conhecimento humano, contribuindo com a tomada de decisões, do ponto de vista prático, por parte dos gestores. Aplicada às variáveis relacionadas ao consumo de água tratada, a metodologia geoestatística permite fazer a análise dos dados, destacando a modelagem da dependência espacial entre as observações e com isso possibilita realizar a interpolação por krigagem e o mapeamento do consumo. Neste mapeamento podem-se destacar, visualmente, as regiões da cidade de maior e de menor consumo, ou seja, permite que ações pontuais possam ser tomadas por parte dos gestores.

A metodologia geoestatística possui aplicação em diversas áreas de conhecimento como, por exemplo, na meteorologia, Loureiro e Fernandes (2013); na agricultura, Faria et al. (2004); na hidrologia e meio ambiente, Castro Junior et al. (2007), entre outras aplicações.

Cabe ressaltar que a cidade de Uberlândia - MG tem experimentado grande desenvolvimento econômico e social nas últimas décadas (CEPES, 2018), colocando-a como uma das maiores cidades do estado, e com isso ocorreu aumento significativo da população e, consequentemente, ocorreu também aumento da demanda de água tratada para o consumo residencial.

Neste sentido, os objetivos deste trabalho foram: analisar e modelar a dependência espacial do consumo médio diário de água tratada, nos meses de janeiro a dezembro do ano de 2015, por meio de semivariogramas; utilizar os modelos ajustados para construir por meio de interpolação por krigagem, os mapas de consumo que descreveram a distribuição espacial do consumo na cidade.

\section{MATERIAL E MÉTODOS}

A cidade de Uberlândia-MG está situada na mesorregião do Triângulo Mineiro e Alto Paranaíba, no estado de Minas Gerais (Figura 1). Em termos populacionais é a segunda cidade mais populosa do estado. A população estimada no ano de 2015 era de 669.672 habitantes (IBGE, 2016). 
Figura 1 - Localização da cidade de Uberlândia - MG.

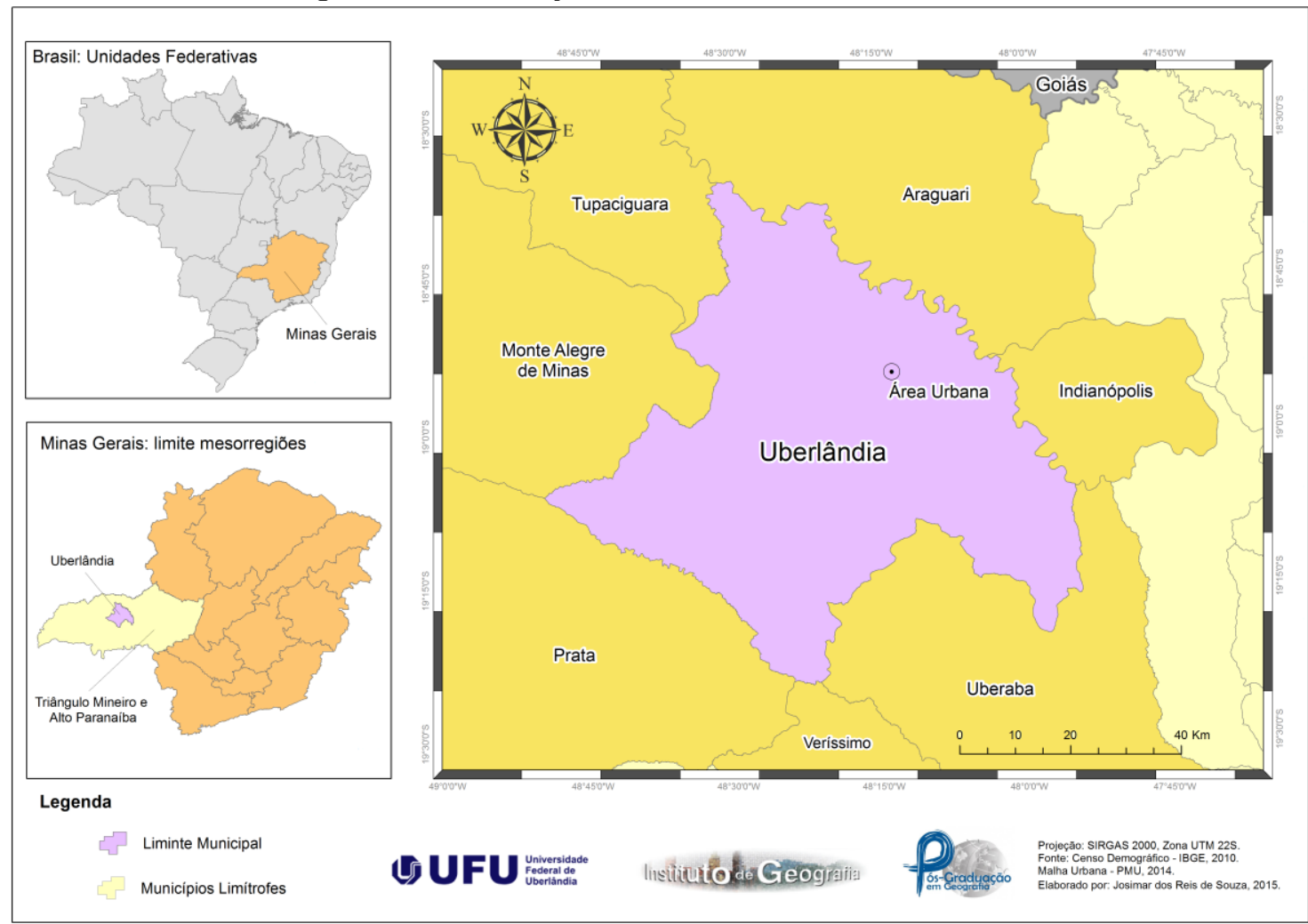

Fonte - Souza (2016).

A variável em análise neste trabalho foi o consumo médio diário $\left(\mathrm{m}^{3}\right)$ de água tratada, nas residências da zona urbana da cidade, sendo esta média avaliada para cada mês do ano de 2015. Os dados foram obtidos junto ao Departamento Municipal de Água e Esgoto (DMAE) de Uberlândia - MG (UBERLÂNDIA, 2020). As informações foram disponibilizadas como o consumo total por bairro em cada mês, associadas ao número de ligações do recurso por bairro no mês, sendo o consumo médio diário no mês por bairro obtido pela relação entre consumo total e número de ligações.

A caracterização geral do consumo médio diário por mês na cidade de Uberlândia foi feita por meio da análise exploratória dos dados e teve a finalidade de verificar o comportamento geral da variável, no período avaliado. Nesta fase de análise foram calculadas as estatísticas (médias, quartis, mínimo, máximo, desvio padrão e coeficiente de variação). Esta análise foi realizada após a verificação inicial das inconsistências do banco de dados.

A dependência espacial foi avaliada por meio do cálculo das semivariâncias dos valores observados, conforme descrito em Vieira et al (1983) e Soares (2014), que geraram os semivariogramas utilizados na análise da estrutura de dependência espacial. As semivariâncias são obtidas pelo estimador de Matheron que é dado pela equação 1:

$$
\gamma(h)=\frac{1}{2 N(h)} \sum_{i=1}^{N(h)}\left[Z\left(x_{i}\right)-Z\left(x_{i}+h\right]^{2}\right.
$$

Em que: $\chi(h)$ é a semivariância para uma distância $h ;\left[Z\left(x_{i}\right), Z\left(x_{i}+h\right)\right]$ são os pares de observações da variável $Z$ nas posições $x_{i}$ e $x_{i+h}$ e $N(h)$ o número de pares na distância $h$.

Os resultados das semivariâncias médias para cada distância $h$ são plotados em um gráfico denominado semivariograma e, com base nestes resultados, são definidos os modelos teóricos que descrevem o comportamento da dependência espacial da variável. 
Para adequação do modelo, foi utilizada a técnica Eyefit do programa de domínio público geoR (Ribeiro Júnior e Diggle, 2001), que consiste em ajustar um modelo inicial e alterar os valores de efeito pepita (Co), contribuição (C) e alcance (a), que são os parâmetros que caracterizam o modelo, até encontrar uma curva que se aproxime melhor dos dados. Dependendo do ajuste obtido, se pode ou não redefinir o modelo, até obtenção de um que seja considerado satisfatório CAMARGO (1998), sendo que a seleção do modelo foi baseada na técnica de validação cruzada.

O modelo estatístico utilizado para o consumo médio diário de água tratada na cidade de Uberlândia, utilizando a técnica de validação cruzada para a seleção do melhor modelo, foi o Gaussiano, definido pela equação 2.

$$
\gamma(h)=C o+C\left[1-\exp \left(-3 \frac{h^{2}}{a^{2}}\right)\right]
$$

Em que: $\not$ (h) é a semivariância para uma distância de separação entre pontos $h, a$ é o alcance da dependência espacial, $C_{0} \circ$ efeito pepita ou variação não estruturada no espaço, $C$ é a contribuição da dependência espacial ou variação estruturada e $\mathrm{C}_{0}+\mathrm{C}$ é o patamar do modelo.

O grau de dependência espacial dos modelos ajustados foi calculado por meio da relação entre $C_{\circ} e$ $\mathrm{C}_{0}+\mathrm{C}$, conforme descrito em Cambardella et al (1994) em que são considerados de dependência espacial forte os semivariogramas que têm efeito pepita $<25 \%$ do patamar, de dependência espacial moderada quando o efeito pepita está entre 25 e $75 \%$ e de dependência espacial fraca, quando o efeito pepita é $>75 \%$.

Definido o modelo do semivariograma, passou-se para a fase de interpolação dos dados. A técnica de interpolação utilizada foi a krigagem. Segundo Ribeiro Junior (1995), krigagem é a estimação de uma matriz de covariância espacial que determina os pesos atribuídos a diferentes amostras, o tratamento da redundância dos dados, a vizinhança a ser considerada no procedimento de inferência e o erro associado ao valor estimador.

O estimador de krigagem é dado pela equação 3 :

$$
Z *(X)=\sum_{i=1}^{k} \lambda_{i} Z\left(x_{i}\right)
$$

Em que: $Z^{*}(X)$ é o valor estimado na posição $X ; \lambda_{i}$ é o peso atribuído ao valor observado da variável $Z$ na posição $\mathrm{X}_{\mathrm{i}}$; $\mathrm{k}$ é o número de vizinhos usados nas estimativas.

Os resultados finais das interpolações por krigagem foram expressos em forma de mapas representativos da variável consumo de água residencial, para a área de estudo, na cidade de Uberlândia-MG, para cada mês do ano de 2015, que possibilitaram a visualização da distribuição espacial desta variável ao longo do tempo.

Todas as análises foram realizadas no pacote geoR (Ribeiro Júnior e Diggle, 2001), software $R$ ( $R$ Core Team, 2017), que são de domínio público.

\section{RESULTADOS E DISCUSSÃO}

Na Tabela 1 são apresentadas as estatísticas do consumo diário de água tratada, nas residências da cidade de Uberlândia, para os meses de janeiro a dezembro do ano de 2015. 
Tabela 1 - Análise descritiva do consumo diário de água tratada $\left(\mathrm{m}^{3}\right)$, nas residências da cidade de Uberlândia-MG, referente ao ano de 2015. (Fonte dos dados primários: DMAE-Uberlândia).

\begin{tabular}{ccccccccccc} 
Meses & Média & Mediana & Q1 & Q3 & Mín & Max & $\mathbf{S}$ & $\mathbf{C V}$ & $\mathbf{S} \mathbf{k}$ & $\mathbf{K}$ \\
\hline Janeiro & 0,6328 & 0,6170 & 0,5420 & 0,7215 & 0,3300 & 0,8980 & 0,1274 & 20,1339 & 0,0773 & $-0,6287$ \\
Fevereiro & 0,5734 & 0,5420 & 0,4820 & 0,6490 & 0,3900 & 0,8950 & 0,1218 & 21,2490 & 0,7415 & $-0,1760$ \\
Março & 0,5930 & 0,5755 & 0,5095 & 0,6617 & 0,4080 & 0,8790 & 0,1145 & 19,3045 & 0,5390 & $-0,4620$ \\
Abril & 0,5870 & 0,5550 & 0,4870 & 0,6630 & 0,4000 & 0,9000 & 0,1287 & 21,9235 & 0,7404 & $-0,4428$ \\
Maio & 0,6004 & 0,5780 & 0,5150 & 0,6710 & 0,4050 & 0,8980 & 0,1196 & 19,9254 & 0,6817 & $-0,2151$ \\
Junho & 0,5873 & 0,5675 & 0,5038 & 0,6560 & 0,4000 & 0,8790 & 0,1162 & 19,7787 & 0,6179 & $-0,3650$ \\
Julho & 0,6132 & 0,5905 & 0,5245 & 0,6847 & 0,4050 & 0,8930 & 0,1205 & 19,6477 & 0,5398 & $-0,3851$ \\
Agosto & 0,6554 & 0,6390 & 0,5790 & 0,7435 & 0,4220 & 0,8910 & 0,1097 & 16,7439 & 0,1367 & $-0,6289$ \\
Setembro & 0,6512 & 0,6485 & 0,5787 & 0,7260 & 0,4150 & 0,8970 & 0,1109 & 17,0349 & $-0,0589$ & $-0,6347$ \\
Outubro & 0,6580 & 0,6535 & 0,5645 & 0,7550 & 0,4030 & 0,8900 & 0,1225 & 18,6176 & 0,0253 & $-0,8232$ \\
Novembro & 0,6389 & 0,6320 & 0,5460 & 0,7250 & 0,3800 & 0,8990 & 0,1214 & 19,0092 & 0,0196 & $-0,7550$ \\
Dezembro & 0,6425 & 0,6390 & 0,5685 & 0,7235 & 0,4000 & 0,8920 & 0,1125 & 17,5061 & 0,0773 & $-0,6287$
\end{tabular}

Q1 = 1o quartil; Q3 = 3o quartil; Min = mínimo; Max = Máximo; S = desvio padrão; CV = coeficiente de variação; $\mathrm{S}_{\mathrm{k}}=$ coeficiente de assimetria; $\mathrm{K}=$ coeficiente de curtose.

Pode-se observar que o mês de outubro se destaca com um maior consumo médio residencial de água e o mês de abril obteve-se o maior coeficiente de variação (CV).

Observa-se ainda por meio da Tabela 1, que os valores de média e de mediana para cada mês estão relativamente próximos, que ocorre uma equidistância aproximada do primeiro e terceiro quartil em relação à mediana e que os coeficientes de assimetria estão relativamente próximos de zero. Estes fatos levam a classificar as distribuições do consumo de água como sendo aproximadamente simétricas. Yamamoto e Landim (2013) descrevem que a aplicação das técnicas de análise geoestatística não necessitam de normalidade dos dados, entretanto, para o uso do estimador de semivariância de Matheron é recomendado que os dados não apresentem assimetria acentuada.

Em relação à variabilidade, medida pelo $\mathrm{CV}$, nota-se que esta tem comportamento semelhante ao longo do ano, levando a induzir que o consumo de água se afasta da média de consumo de forma semelhante ao longo dos meses. Mas o CV não avalia a dispersão espacial e então, apesar da distribuição em torno da média ser parecida, pode ocorrer comportamento espacial diferenciado para os meses do ano e que deve ser avalido por meio dos semivariogramas.

A análise do semivariograma para cada mês do ano de 2015 permitiu ajustar o modelo teórico do tipo gaussiano em todas as situações, conforme mostram os gráficos da Figura 2. A escolha do modelo, assim como as estimativas dos parâmetros do modelo foi feita com base na técnica de validação cruzada.

A seleção do modelo gaussiano para descrever o comportamento espacial do consumo de água tratada na cidade de Uberlândia, nos meses de janeiro a dezembro, mostra que o padrão espacial é semelhante ao longo do tempo, entretanto, o fato dos modelos ajustados não apresentarem estimativas dos parâmetros semelhantes ou proporcionais entre sí não permite dizer que ocorre a estabilidade temporal, ou seja, o comportamento espacial não é afetado apenas pela escala de consumo ao longo do tempo, mas também pelos hábitos de consumo de cada bairro. 
Figura 2 - Semivariogramas experimentais com modelos gaussianos ajustados por mês para o ano de 2015.
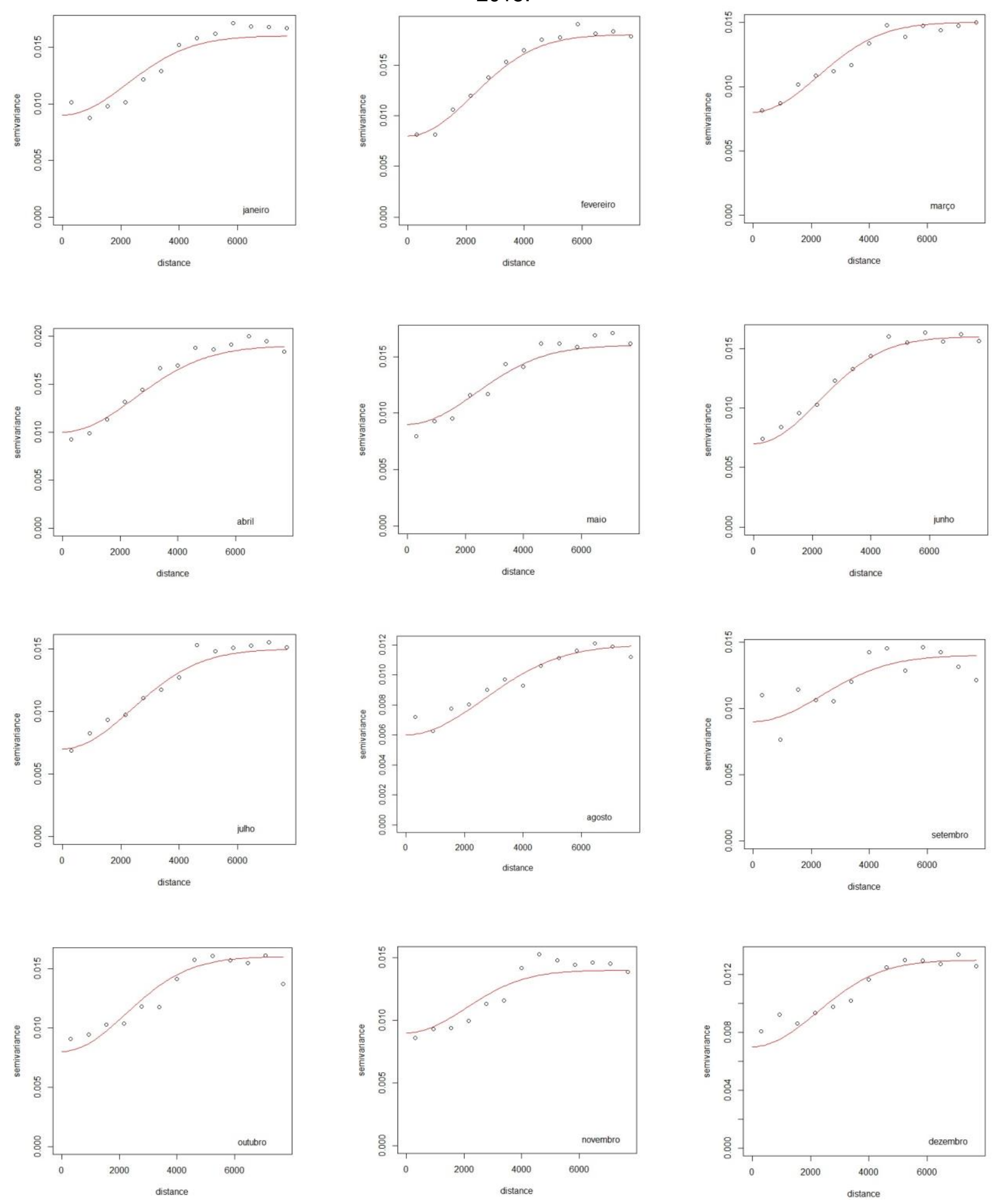

Na Tabela 2 são apresentadas as estimativas dos parâmetros dos modelos gaussianos ajustados assim como os respectivos graus de dependência. 
Tabela 2 - Estimativas dos parâmetros: Efeito pepita (Co), Contribuição (C) e alcance (a), para os modelos gaussianos e grau de dependência (GD) do consumo de água tratada nas residências da zona urbana de Uberlândia - MG, por mês para o ano de 2015.

\begin{tabular}{ccccc}
\hline Meses & CO & C & a & GD \\
\hline Janeiro & 0,0090 & 0,0070 & 5397,2110 & 0,5625 \\
Fevereiro & 0,0080 & 0,0100 & 5397,2110 & 0,4444 \\
Março & 0,0080 & 0,0070 & 5397,2110 & 0,5333 \\
Abril & 0,0100 & 0,0090 & 6116,8333 & 0,5263 \\
Maio & 0,0090 & 0,0070 & 5757,0135 & 0,5625 \\
Junho & 0,0070 & 0,0090 & 5397,2110 & 0,4375 \\
Julho & 0,0070 & 0,0080 & 5757,0135 & 0,4667 \\
Agosto & 0,0060 & 0,0060 & 6476,6359 & 0,5000 \\
Setembro & 0,0090 & 0,0050 & 5757,0135 & 0,6429 \\
Outubro & 0,0080 & 0,0080 & 5397,2110 & 0,5000 \\
Novembro & 0,0090 & 0,0050 & 5037,3911 & 0,6429 \\
Dezembro & 0,0070 & 0,0060 & 5397,2110 & 0,5385 \\
\hline
\end{tabular}

A Tabelas 2 mostra que, considerando a classificação proposta por Cambardella et al. (1994), ocorreu dependência espacial de grau moderado para todos os meses pois os GD ficaram entre 0,25 e 0,75 .

O alcance da dependência mínimo encontrado foi de 5037,3911UTM para o mês de novembro e o máximo foi de 6476,6359 UTM para o mês de agosto. Portanto, existe uma correlação espacial, para os consumos de água tratada, entre as residências que se encontram afastadas entre si em círculos com raios que variam na magnitude dos alcances associados a cada mês. Este fato pode contribuir com o planejamento de uso, distribuição e consumo de água por parte dos gestores do DMAE, pois se ocorre a dependência espacial é possível mapear o consumo por região, considerando-se faixas pré-estabelecidas, desta forma pode-se visualizar as regiões onde ocorrem maiores e menores consumos de água.

Com base nos semivariogramas ajustados, as interpolações usando a krigagem ordinária foram realizadas. A metodologia de krigagem ordinária assume que os valores na região de interesse não apresentam tendência que possam afetar os resultados e possibilitaram a confecção dos mapas, para cada mês do ano de 2015. Estes expressam áreas com maior ou menor consumo de água tratada na zona urbana de Uberlândia-MG, conforme apresentado na Figura 3, para a zona urbana da cidade.

Os resultados da Figura 3 apontam uma tendência de padrão de concentração de maior consumo na região central do mapa representativo da área urbana da cidade, sendo que, para a cidade de Uberlândia, esta área representa o bairro denominado Centro e seus respectivos bairros limítrofes.

A expansão urbana da cidade tem ocorrido nas regiões periféricas do mapa representativo e apresenta, no geral, os menores consumos de água. Este fato pode estar associado a conscientização de novos empreendimentos imobialiários em relação a importância do uso desse recurso, fazendo com que se busque equipamentos domésticos que minimizem o uso da água, assim como formas de reuso da água ou armazenamento de água de chuva para uso em determinadas atividades residenciais. 
Figura 3 - Mapeamento por krigagem da variável consumo residencial de água tratada na zona urbana da cidade de Uberlândia - MG, nos meses de janeiro a dezembro de 2015.
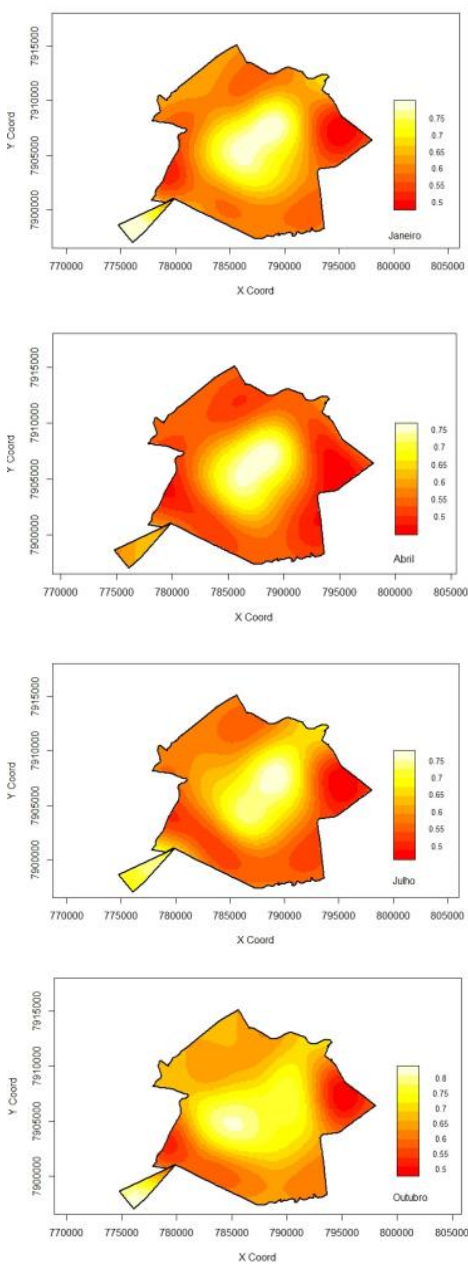
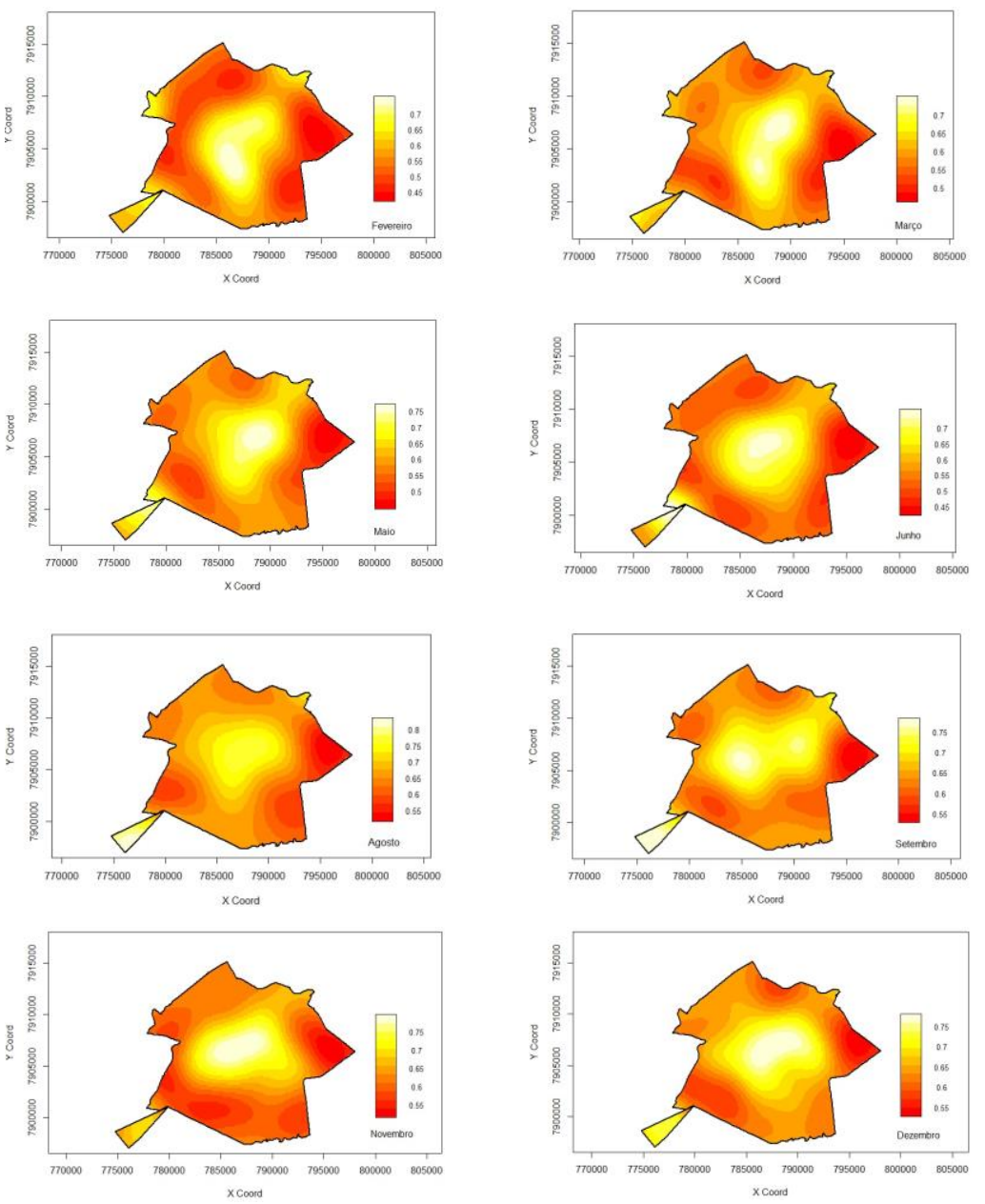

Observando os mapas gerados para os meses de janeiro a dezembro de 2015, nota-se que o padrão espacial é semelhante para todos os meses. Isto se deve ao fato do ajuste do modelo espacial gaussiano para todos os meses. Entretanto, percebem-se diferenças nas áreas e na disposição das faixas de consumo. Estas diferenças são devidas as variações que ocorreram no alcance e na contribuição da dependência espacial para cada mês. Por exemplo, ao se comparar os mapas dos meses de agosto e novembro, que apresentaram, respectivamente, o maior e o menor alcance da dependência espacial e também graus de dependência diferenciados, nota-se que não há semelhança nas distribuições das faixas de ocorrência de consumo, reforçando a ideia de que os hábitos de consumo de água ao longo dos meses do ano não é semelhante entre os diversos bairros da cidade.

Um aspecto interessante para a análise de uma variável ao longo do tempo, se refere a estabilidade temporal, ou seja, verificar se a variabilidade espacial da variável em análise é a mesma ao longo do tempo, variando-se apenas a escala dessa variabilidade. Autores como Gonçalves et al. (1999) e Salvador et al. (2012) abordaram aspectos da estabilidade temporal da variabilidade espacial e argumentaram que, se a estabilidade temporal ocorre um modelo de dependência padronizado pode ser utilizado para se fazer as estimativas em qualquer tempo e que para a geração dos mapas os valores obtidos na interpolação devem ser multiplicados pelo fator de escala de cada tempo. Este fato pode contribuir com a redução no tempo de análise dos dados e facilitar tomadas de decisões, pois faixas com valores relativos maiores ou menores tendem a apresentar este aspecto em qualquer época de análise. Cabe ressaltar que, quando ocorre a estabilidade temporal o fator de escala, que padroniza o modelo de dependência, é dado pela variância dos dados. 
Neste estudo, apesar dos modelos ajustados terem sido sempre o gaussiano, verificou-se diferenças nos parâmetros do modelo, ou seja, diferentes alcances e contribuições da dependência espacial, portanto não ocorre estabilidade temporal entre os meses do ano. Um estudo futuro pode ser realizado para verificar se esta estabilidade temporal ocorre para cada mês em anos diferentes.

Trabalhos e estudos científicos que envolvem a ferramenta geoestatística na determinação da dependência espacial e no mapeamento das variáveis de interesse são comuns em áreas de estudos envolvendo agricultura, meio ambiente, mineração, hidrologia, climatologia, dentre outras, entretanto, não se conseguiu relatos de trabalhos que envolvam abastecimento ou consumo de água tratada em cidades usando essa técnica, impossibilitando a comparação e a discussão dos resultados aqui obtidos com aqueles advindo da literatura.

\section{CONSIDERAÇÕES FINAIS}

Ressalta-se a eficiência da geoestatística voltada para o estudo da variável analisada, pois foi observada a dependência espacial em todas as situações estudadas. Portanto, os mapas gerados por meio de técnicas geoestatística irão produzir estimativas mais precisas do que aqueles gerados por técnicas de interpolação que não consideram a dependência espacial descrita pelo semivariograma.

A geoestatística consiste em uma ferramenta inovadora, por permitir a espacialização dos dados e impor uma maior precisão aos estudos relacionados a esse tema, pois além de considerar o valor do consumo em si, considera também a distribuição no espaço e seu grau de relação com os vizinhos.

O mapeamento feito por meio da krigagem, quando comparado com outros tipos de interpoladores, permite realizar estimativas com maior precisão e desta forma irá produzir um panorama mais realístico do consumo de água tratada na cidade de Uberlândia - MG e medidas, como a de conscientização sobre o uso de água tratada, podem ter focos específicos para diferentes áreas urbanas da cidade.

\section{AGRADECIMENTOS}

O presente trabalho foi realizado com apoio da Coordenação de Aperfeiçoamento de Pessoal de Nível Superior - Brasil (CAPES) - Código de Financiamento 001 e do Programa Institucional de Bolsas de Iniciação Científica (PIBIC/CNPq/UFU).

\section{REFERENCIAS}

ARAÚJO, M. F. C.; GUIMARÃES, E. C.; CARVALHO, D. F. ; ARAÚJO, L. B. Precipitação pluviométrica mensal no Estado do Rio de Janeiro: sazonalidade e tendência. Bioscience Journal, Uberlândia, v. 25, n. 4, p.90-100, ago. 2009.

CAMARGO, E. C. G. Geoestatística: fundamentos e aplicações. In: CAMARA, Gilberto; MEDEIROS, J. S. de. Geoprocessamento para Projetos Ambientais. São José dos Campos: INPE, 1998. Cap. 5. p. 1-36.

CAMBARDELLA, C.A.; Moorman, T.B; Novack, J.M; Parkin, T.B; Karlen, D.L; Turco R.F.; Knopka, A.E. Field-scale variability of soil proprieties in central lowa soils. Soil Science Society America $\begin{array}{llll}\text { Journal, Medison, } & \text { v.58, } & \text { p.1240-1248, } & 1994 .\end{array}$ https://doi.org/10.2136/sssaj1994.03615995005800050033x

CASTRO JUNIOR, R. M.; SOBREIRA, F. G.; BORTOLOTI, F. D. Modelagem geoestatística a partir de parâmetros de qualidade da água (IQA-NSF) para a sub-bacia hidrográfica do Rio Castelo (ES) usando sistema de informações geográficas. Revista Brasileira de Cartografia, Ouro Preto, v. 59, n. 3, p.241-253, 2007.

CEPES: Centro de Estudos, Pesquisas e Projetos Econômicos-socias . Uberlândia - Painel de Informações Municipais 2018. Uberlândia-MG: Centro de Estudos, Pesquisas e Projetos Econômicosociais/Instituto de Economia e Relações Internacionais/Universidade Federal de Uberlândia, agosto 2018. 93 p. Disponível em: http://www.ie.ufu.br/CEPES. Acesso em: 05/12/2018

FARIA, N. M. X.; FACCHINI, L. A.; FASSA, A. G.; TOMASI, E. Trabalho rural e intoxicações por 
agrotóxicos. Cadernos de Saúde Pública, v. 20, n. 5, p. 1298-308, set./out. 2004 https://doi.org/10.1590/S0102-311X2004000500024

GONÇALVES, A. C. A.; FOLEGATTI, M. V.; SILVA, A. P. Estabilidade temporal da distribuição espacial da umidade do solo em área irrigada por pivô central. Revista Brasileira de Ciência do Solo,

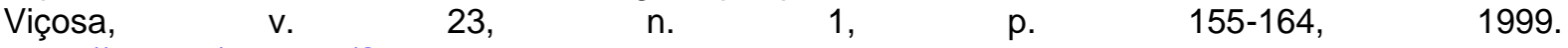
https://doi.org/10.1590/S0100-06831999000100019

HESPANHOL, I. Água e saneamento básico - Uma visão realista. In: REBOUÇAS, A. et al (org.) Águas Doces no Brasil. (São Paulo: Instituto de Estudos Avançados da USP, 1999.

IBGE: Instituto Brasileiro de Geografia e Estatística. Minas Gerais: Uberlândia, MG, 2016. Disponível em: < https://cidades.ibge.gov.br/brasil/mg/uberlandia/panorama>. Acesso em: 05/12/2018

LOUREIRO, G. E.; FERNANDES, L. L.. Variação da precipitação por método de interpolação geoestatística. Revista Ambiente \& Água, Taubaté, v. 8, n. 2, p.77-87, 2013.

PINTO, W. de P.; LIMA, G. B.; ZANETTI, J. B. Análise comparativa de modelos de séries temporais para modelagem e previsão de regimes de vazões médias mensais do Rio Doce, Colatina - Espírito Santo. Revista do Centro de Ciências Naturais e Exatas, Santa Maria, v. 37, n. 4, p.1-11, ago., 2015. https://doi.org/10.5902/2179460X17143

R CORE TEAM . R: A language and environment for statistical computing. Vienna: R Foundation for Statistical Computing, 2017.

RIBEIRO JUNIOR, P. J.; DIGGLE, P. J. GeoR: a package for geostatistical analysis. R News, London, v. 1, n. 2, p. 15-18, 2001.

RIBEIRO JUNIOR, P. J. Métodos geoestatísticos no estudo da variabilidade espacial de parâmetros de solo. Dissertação (Mestrado em Estatística e Experimentação Agronômica) - Escola Superior de Agricultura Luiz de Queiroz, Universidade de São Paulo, Piracicaba, 1995.

SALVADOR, M. M. S.; LIBARDI, P. L.; BRITO, A. S.; MOREIRA, N. B. Estabilidade temporal e variabilidade espacial da distribuição de armazenagem de água no solo numa sucessão feijão/aveiapreta. Revista Brasileira de Ciência do Solo, Viçosa, v. 36, n. 5, p. 1434-1447, 2012. https://doi.org/10.1590/S0100-06832012000500007

SOARES, A. Geoestatística Para as Ciências da Terra e do Ambiente, IST Press, 232 p., 2014.

SOUZA, J. R. Trilhando por cidades saudáveis: contribuição metodológica de índice e aplicação em Uberlândia, MG. (Mestrado em Geografia) - Instituto de Geografia, Universidade Federal de Uberlândia, Uberlândia, 2016.

UBERLÂNDIA (município). DMAE: Departamento Municipal de Água e Esgoto. O DMAE. Disponível em: https://www.uberlandia.mg.gov.br/prefeitura/orgãos-municipais/dmae. Acesso em: 10/02/2020.

VIEIRA, S.R.; HATFIELD, J.L.; NIELSEN, D.R. \& BIGGAR, J.W. Geoestatistical theory and application to variability of some agronomical properties. Hilgardia, Berkeley, 51(3):1-75, 1983. https://doi.org/10.3733/hilg.v51n03p075

YAMAMOTO, J.K.; LANDIM, P.M.B. Geoestatística: conceitos e aplicações. São Paulo: Oficina de textos, 2013. 215p.

Recebido em: 11/10/2019

Aceito para publicação em: 10/02/2020 